\title{
ADIABATIC THEORY OF PARTICLE TRAPPING DUE TO COULOMB CROSSING OF ONE-DIMENSIONAL BETATRON RESONANCE
}

\author{
P. Zenkevich \\ Institute of Theoretical and Experimental Physics, Moscow \\ I. Hofmann, G. Franchetti \\ GSI, Darmstadt, Germany
}

In the presence of the space charge (s.c.) forces the synchrotron oscillations result in periodical modulation of the s.c. tune shift, periodical crossing of betatron resonances and particle trapping in resonance islands. The trapping effect for one-dimensional resonance is considered using classical perturbation theory and «frozen core» approach to calculation of the s. c. forces. The beam losses and the emittance growth are analyzed for the arbitrary order resonance; the numerical results are given for the third-order resonance.

Численное моделирование поперечных резонансных эффектов в присутствии пространственного заряда [1] показало, что благодаря этим эффектам частицы могут захватываться в стабильные резонансные острова. Мы анализируем влияние сил пространственного заряда на пересечение одномерного резонанса, используя классическую теорию возмущений и метод «замороженного ядра» для расчета кулоновских сил. Выведена простая формула, определяющая зависимость нелинейного кулоновского сдвига бетатронных частот от амплитуды поперечных колебаний для мягкой фокусировки и круглого пучка. Анализ стабильных сепаратрис поперечных колебаний в присутствии кулоновского сдвига бетатронных частот показал, что октупольная нелинейность магнитного поля при правильном выборе знака уменьшает амплитуды колебаний захваченных частиц. Найден простой алгоритм оценки потерь пучка и роста эмиттанса для резонанса произвольного порядка в процессе захвата частиц при модуляции бетатронной частоты, вызванной синхротронными колебаниями. Сравнение с результатами численного моделирования для резонанса третьего порядка подтвердило применимость теории возмущений к проблеме кулоновского пересечения резонанса.

PACS: 29.20.df

\section{INTRODUCTION}

Let us consider particle dynamics in circular accelerators and storage rings in the presence of the space charge (s.c.) fields and the external perturbations creating one-dimensional betatron resonance. A numerical modeling for the third-order resonance [1] has shown that due to interaction of these effects the particles may be trapped into the stable islands.

Assuming that main part of the beam is unperturbed, we can use for the analytic studies so-called «frozen core» model [2]. A theory of the particle motion near the isolated nonlinear 
resonance is developed in papers $[3,4]$ using the canonical «action-phase» variables $J, \phi$; specifics of our effect is connected with special form of the nonlinear tune dependence.

In bunched beams the space charge linear density (and consequently the «instantaneous» s.c. shift) depends on longitudinal coordinate of the particle inside the bunch; therefore, for some values of unperturbed betatron tune the betatron resonance is crossed periodically due to the synchrotron oscillations.

The perturbation theory shows that in the presence of nonlinear tune dependence the particles captured in the stationary separatrix oscillate around its center. For narrow nonlinear resonance these oscillations are characterized by the following parameters: 1 ) $J_{0}$ (value of action corresponding to the separatrix center); 2) $\Omega_{s}$ (frequency of the particle linear oscillations around the separatrix center); 3) $\Delta J_{s}$ (the separatrix width). If the motion is nonstationary, the particles cross the resonance line. A character of the process depends on the «adiabaticity parameter» $K_{\mathrm{ad}}=\frac{\delta J_{s}}{\Delta J_{s}}$ where $\delta J_{s}$ is a shift of $J_{0}$ during the period of the particle oscillations around the separatrix center. This process is examined by A. Chao [5] for the particular case of the fifth-order one-dimensional resonance. An estimate of the «trapping efficiency» is made in assumption that all particles with $K_{\text {ad }} \leqslant 1$ are trapped and all particles with $K_{\mathrm{ad}}>1$ are nontrapped. Here we use the same criterion and assume that all particles crossing the resonance have $K_{\mathrm{ad}} \leqslant 1$ («adiabatic model») and, consequently, are captured in the nonlinear separatrix.

For generalization we consider a resonance of an arbitrary order; the numerical results are applied to the third-order resonance. Let us mark that the adiabatic model gives only the «upper limit» for the losses; nevertheless, it can be useful due to its simplicity and common theory for arbitrary order resonances.

\section{PARAMETERS OF STABLE ISLANDS}

Initial Assumptions. Let us assume that transverse motion in horizontal plane $(y=0)$ is defined by the following equation:

$$
\ddot{x}+\left(\frac{Q_{0}}{R}\right)^{2} x=\frac{e Z_{i}}{A_{i} m_{p} c^{2} \beta^{2} \gamma^{3}} E(x) \exp \left(-\frac{z^{2}}{2 \sigma_{z}^{2}}\right)+a_{n} \delta(s) x^{n-1} .
$$

In Eq. (1) $x$ is the transverse coordinate of the particle; $z$ is the particle distance from the bunch center; independent variable $s$ is a length along the ring circumstances; $Q_{0}$ is the unperturbed betatron tune; $e$ is electron charge; $m_{p}$ is proton mass; $\beta, \gamma$ are relativistic parameters; $Z_{i}$ and $A_{i}$ are, correspondingly, charge and atomic numbers of circulating particle; $E(x)$ is the beam electrical field at the bunch center in the rest system; $\delta(s)$ is delta function; $a_{n}$ is an amplitude of the nonlinear lens. Here we consider axe-symmetric Gaussian beam with space charge density $\rho(x, y, z)$ proportional to $\exp \left(-\frac{x^{2}+y^{2}}{2 \sigma_{x}^{2}}-\frac{z^{2}}{2 \sigma_{z}^{2}}\right)$. In Eq. (1) $\sigma_{x}, \sigma_{y}, \sigma_{z}$ are corresponding rms beam sizes $\left(\sigma_{x}=\sigma_{y}\right), \sigma_{i}=\sqrt{\varepsilon_{i} \beta_{i}}\left(\varepsilon_{i}\right.$ are rms emittances, $\beta_{i}$ are the corresponding beta functions).

In paper [1] the third-order resonance $(n=3)$ is considered. For numerical analysis parameters of the SIS-18 synchrotron were taken: machine radius $R=34.4 \mathrm{~m}$, horizontal synchrotron tune $Q_{0}=4.3, \sigma_{x}=0.01 \mathrm{~m}$. For round Gaussian beam the s.c. force in the 
right-hand side (RHS) of Eq. (1) is

$$
\text { RHS }=K \exp \left(-\frac{z^{2}}{\sigma_{z}^{2}}\right) \frac{1}{x}\left[1-\exp \left(-\frac{x^{2}}{\sigma_{x}^{2}}\right)\right] .
$$

Perveance $K$ is connected with linear Coulomb shift $\Delta Q_{\mathrm{sc}}^{0}$ by the following formula: $K=$ $\Delta Q_{\mathrm{sc}}^{0} \frac{2 \sigma^{2}}{R^{2}}\left(2 Q_{0}-\Delta Q_{\mathrm{sc}}^{0}\right)$. For SIS-18 we obtain the resonance width $g \sim 10^{-4}$. Let us note that in the following text we use normalized dimensionless invariant $J=\varepsilon / \varepsilon_{0}$.

Hamiltonian and Equations of Motion. A value of the nonlinear s.c. tune shift $\Delta Q_{\mathrm{sc}}$ due to beam-beam interaction was derived in paper [4]. Generalizing this formula for one beam s.c. interaction, we find that $\Delta Q_{\mathrm{sc}}=\Delta Q_{\mathrm{sc}}^{0} \Phi_{\mathrm{sc}}(J)$, where function

$$
\Phi_{\mathrm{sc}}(J)=\frac{2}{J}\left[1-\exp \left(-\frac{J}{2}\right) I_{0}\left(\frac{J}{2}\right)\right] .
$$

Here $I_{0}(x)$ is modified Bessel function of the zeroth order. Using the perturbation theory we can write the one-dimensional Hamiltonian in variables $J, \phi$ (action-phase) for the arbitrary resonance order $n$ (taking into account only the third order of external nonlinearity) in the following form:

$$
\delta H=\Delta \nu_{\mathrm{sc}}^{0} \Psi(J)+K \frac{J^{2}}{2}-\frac{2}{n} J^{n / 2} \cos (n \phi) .
$$

Here as an independent variable we use «slow angle» $\theta=g s / R$; all parameters are divided by the resonance strength $g\left(\Delta \nu_{0}=\Delta Q_{x}^{0} / g, \Delta \nu_{\mathrm{sc}}^{0}=\Delta Q_{\mathrm{sc}}^{0} / g, K=k / g\right)$. Function $\Psi(J)$ is defined by the equation $\Phi_{\mathrm{sc}}(J)=\frac{d \Psi(J)}{d J}$. The canonical equations of motion are

$$
\begin{aligned}
& \frac{d J}{d \theta}=2 J^{n / 2} \sin (n \phi), \\
& \frac{d \phi}{d \theta}=\Delta \nu_{0}-\Delta \nu_{\mathrm{sc}}^{0} \Phi_{\mathrm{sc}}(J)+K J-J^{\frac{n}{2}-1} \cos (n \phi) .
\end{aligned}
$$

The phase space topology depends on four parameters: two dimensionless tune shifts $\Delta \nu_{0}$, $\Delta \nu_{\mathrm{sc}}^{0}$, the octupole nonlinear coefficient $K$ and order of the resonance $n$. Stationary points are defined by equations $\frac{d J}{d \theta}=0, \frac{d \phi}{d \theta}=0$. Then we find from the equation for $\frac{d J}{d \theta}: \sin (n \phi)=0$, $\cos (n \phi)= \pm 1$. Substituting these values in the Hamiltonian (Eq. (4)) and an equation for $\frac{d \phi}{d \theta}$ (the second line of Eq. (5)), we obtain

$$
\begin{gathered}
H_{ \pm}(J)=\Delta \nu_{0} J-\Delta \nu_{\mathrm{sc}}^{0} \Psi(J) \pm \frac{2}{n} J^{n / 2}+K \frac{J^{2}}{2}, \\
\Delta \nu_{1,2}(J)=\Delta \nu_{0}-\Delta \nu_{\mathrm{sc}}^{0} \Phi_{\mathrm{sc}}(J) \pm \frac{2}{n} J^{\frac{n}{2}-1}+K J .
\end{gathered}
$$

The stable fixed point $J_{0}$ is defined by a solution of the nonlinear equation $\Delta \nu_{2}(J)=0$. The separatrix parameters can be found by standard procedure [3,4] using Eqs. (5)-(7).

Let us consider the equation for the separatrix center $\Delta \nu_{2}\left(J_{0}\right)=0$. Firstly, let us note that in high currents machines the resonance strength $g \ll \Delta Q_{\mathrm{sc}}^{0}$ (for example, in our numerical 
simulations [1] $g=10^{-4}, \Delta Q_{\mathrm{sc}}^{0}=0.1$ and normalized s. c. tune shift $\left.\Delta \nu_{\mathrm{sc}}^{0}=1000\right)$. Then at the first approximation we may neglect resonant term, and an equation for the separatrix center is written as follows:

$$
\Lambda-\Phi_{\mathrm{sc}}(J)+\widetilde{K} J=0 .
$$

Here $\Lambda=\Delta \nu_{0} / \Delta \nu_{\mathrm{sc}}^{0}, \widetilde{K}=K / \Delta \nu_{\mathrm{sc}}^{0}(\Lambda<1$ since it is a condition of resonance crossing). We see that the equation does not depend on the resonance order and the resonance strength!

Let us find an approximate solution for $\widetilde{K}=$ 0 , taking into account that for $J \gg 1 \Phi_{\mathrm{sc}}(J) \rightarrow$ $2 / J$. Then the approximate solution of Eq. (8) is $J_{0} \sim 2 / \Lambda=2 \frac{\Delta Q_{\mathrm{sc}}^{0}}{\Delta Q_{0}}$. We see that the value of invariant for the island center goes to infinity when distance from the resonance goes to zero.

The exact separatrix parameters were found using the program written in MATHCAD language. An example of the results is given in Fig. 1. We see from Fig. 1 that the positive sign of the nonlinearity increases the stability (the area of the separatrix is decreased with $\widetilde{K}$ ).

Theory of Small Oscillations and Adiabaticity Criterion. For narrow nonlinear resonance the oscillations are characterized by the following parameters: 1) $J_{0}$ (value of action corresponding

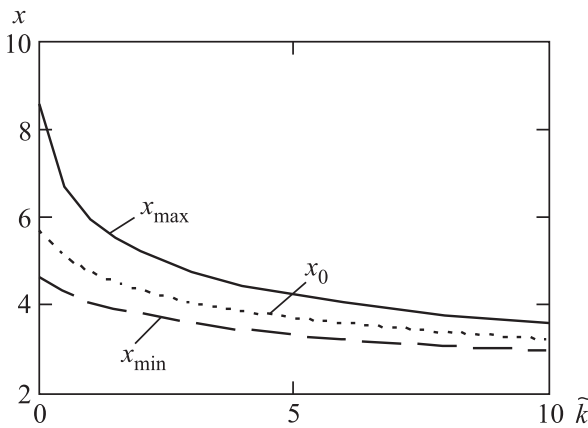

Fig. 1. Dependence of the separatrix parameters $x_{\min }=\sqrt{J_{\min }}, x_{0}=\sqrt{J_{0}}, x_{\max }=$ $\sqrt{J_{\max }}$ on the nonlinearity parameter $(n=3$, $\left.\Delta \nu_{\mathrm{sc}}^{0}=1000, \Delta \nu_{0}=50, \Lambda=1 / 20\right)$ to the separatrix center); 2) $\Omega_{s}$ (frequency of the particle linear oscillations around the separatrix center); 3) $\Delta J_{s}$ (the separatrix width). These parameters can be found using the technique given in [5]. Let us present the Hamiltonian $H(J, \phi)$ in the following form:

$$
H(J, \phi)=H_{0}(J)-\frac{2}{n} J_{0}^{n / 2} \cos (n \phi) .
$$

Here $H_{0}(J)=\Delta \nu_{0} J-\Delta \nu_{\mathrm{sc}}^{0} \Psi(J)+K \frac{J^{2}}{2}$. Near the thin one-dimensional resonance we can expand the function $H_{0}(J)$ in Taylor series on the small parameter $\Delta J=J-J_{0}$; then we can write $H(J, \phi)$ in a form of the pendulum Hamiltonian:

$$
H(J, \phi)=-\frac{1}{2} G\left(J_{0}\right)(\Delta J)^{2}+F\left(J_{0}\right) \cos (\chi) .
$$

Here $\chi=n \phi, G\left(J_{0}\right)=-n \frac{\partial^{2} H_{0}(J)}{\partial J^{2}}\left(J=J_{0}\right)=n \Delta \nu_{\mathrm{sc}}^{0} \frac{d \Phi(J)}{d J}\left(J=J_{0}\right)+K, F\left(J_{0}\right)=$ $2\left(J_{0}\right)^{n / 2}$. The corresponding equations of motion are

$$
\frac{d(\Delta J)}{d \theta}=F\left(J_{0}\right) \sin (\chi), \quad \frac{d \chi}{d \theta}=G\left(J_{0}\right) \Delta J .
$$

This approximation is valid only for thin resonance $\left(\Delta J_{s} / J_{0} \ll 1\right)$. The separatrix width is

$$
\Delta J_{s}=2 \sqrt{\frac{F\left(J_{0}\right)}{G\left(J_{0}\right)}} .
$$


Thus, the criterion of «narrow separatrix» is $K_{\mathrm{ns}}=\frac{2}{J_{0}} \sqrt{\frac{F\left(J_{0}\right)}{G\left(J_{0}\right)}} \ll 1$. For large $J_{0}-\frac{d \Phi}{d J}(J=$ $\left.J_{0}\right) \sim \frac{2}{J_{0}^{2}}$; then we obtain the following estimate for the separatrix width:

$$
\Delta J_{s}=2 \sqrt{2 / n}\left(J_{0}\right)^{n / 4+1}\left(2 \Delta \nu_{\mathrm{sc}}^{0}+K J_{0}^{2}\right) .
$$

We see from Eq. (13) that the separatrix area is increased with growth of the coordinate of the invariant center $J_{0}$. A frequency of the linear oscillations in the island is

$$
\Omega_{s}=\sqrt{F\left(J_{0}\right) G\left(J_{0}\right)} .
$$

\section{ADIABATIC RESONANCE CROSSING IN BUNCHED BEAM}

Adiabatic Island Parameters. In the bunched beam the linear s.c. density and the corresponding tune shift periodically oscillate with the synchrotron frequency; that results in crossing of the resonance «islands». When the island crossing is «adiabatic», a particle can be trapped into the island if the island area $S$ is such that it increases during the passage. According to the theory, a particle remains trapped until the island area returns back to the same value where the original trapping occurs. The typical example of the trapped particle trajectory is given in Fig. 2.
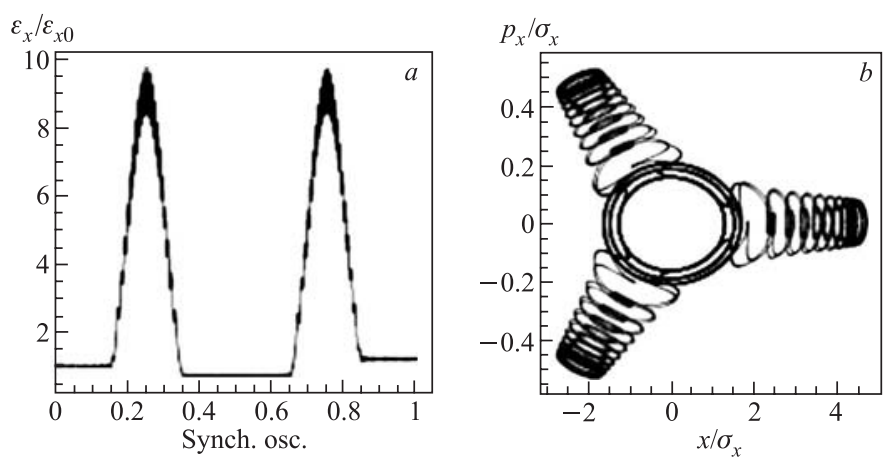

Fig. 2. Trapping of particle during one synchrotron oscillation: $a$ ) single particle invariant; $b$ ) phase space (in normalized units)

In adiabatic approximation the parameters of the captured island coincide with stationary ones. For Gaussian bunch the s.c. tune shift is

$$
\Delta \nu_{\mathrm{sc}}(u)=\Delta \nu_{\mathrm{sc}}^{0} \exp \left(-\frac{u^{2}}{2}\right) .
$$

Here $\nu_{s}=\sqrt{Q_{s} / g}, u=z / \sigma_{s}, z$ is deviation from the bunch center. For $K$ the separatrix characteristics depend on resonance number $n$ and three normalized parameters: space charge 
tune shift $\Delta \nu_{\mathrm{sc}}^{0}$, tune distance from the resonance $\Delta \nu_{0}$ and longitudinal variable $u$. Numerical simulations data $\left(n=3, \Delta Q_{\mathrm{sc}}^{0}=0.1, \Delta Q_{0}=1 / 60, g=10^{-4}\right)$ correspond to the following normalized variables: $\Delta \nu_{\mathrm{sc}}^{0}=1000, \Delta \nu_{0}=167$. Dependence of the separatrix parameters on $u$ is plotted in Fig. 3 (the picture coincides with results of the numerical simulations). We see from Fig. 3 that the separatrix disappears if $u \geqslant u_{t}$, where $u_{t}$ is determined by condition $\Delta \nu_{0}=\Delta \nu_{\mathrm{sc}}^{0} \exp \left(-\frac{u_{t}^{2}}{2}\right) ;$ then

$$
u \quad u_{t}=\sqrt{2 \ln \left(\frac{\Delta \nu_{\mathrm{sc}}^{0}}{\Delta \nu_{0}}\right)}=2 \sqrt{2 \ln \left(\frac{1}{\Lambda}\right)} .
$$

Near $u_{t}$ the separatrix center is defined by the following relation: $J_{0}=\frac{3}{8}\left(u_{t}-u\right)$.

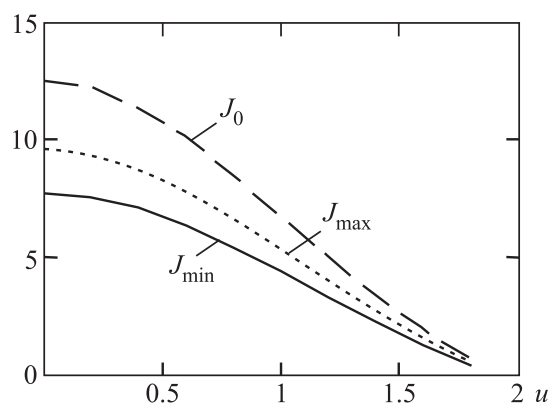

Fig. 3. Dependence of the invariants of the separatrix characteristic points on the normalized longitudinal coordinate $u\left(n=3, \Delta \nu_{\mathrm{sc}}^{0}=1000, \Delta \nu_{0}=167\right)$; dotted curve corresponds to the separatrix center

Adiabaticity Criterion. If the motion is nonstationary, the particles cross the resonance line. A character of the process depends on the «adiabaticity parameter» $K_{\mathrm{ad}}=\delta J_{0} / J_{0}$, where $\delta J_{0}$ is a shift of $J_{0}$ during the period of the particle oscillations around the separatrix center. Let us assume that $J_{0}=J_{0}(u), u$ is a slow changing parameter depending on independent variable $\theta$. Change of $J_{0}$ during one period of transverse oscillations inside the separatrix

$$
\delta J_{0}=\frac{d J_{0}}{d u} \frac{d u}{d \theta} \frac{2 \pi}{\Omega_{s}}=\frac{d J_{0}}{d u} \frac{d u}{d \theta} \frac{2 \pi}{\sqrt{F\left(J_{0}\right) G\left(J_{0}\right)}} .
$$

Then the adiabaticity criterion is

$$
K_{\mathrm{ad}}=\frac{\delta J_{0}}{J_{0}}=\frac{d J_{0}}{d u} \frac{d u}{d \theta} \frac{\pi}{F\left(J_{0}\right)} .
$$

Neglecting the separatrix width, we suppose that $J_{0}=J$, where $J$ is particle invariant. Then we obtain the following condition of the resonance crossing (see Fig. 3):

$$
u_{0} \geqslant u_{1}(J)=u_{t}\left(1-\frac{3}{8 u_{t}} J\right)
$$


Strictly speaking, condition of the adiabaticity is $K_{\text {ad }} \ll 1$; however, here we will use Chao's condition [5] $\left(K_{\mathrm{ad}} \leqslant 1\right)$ to determine the capture area. Then we obtain a necessary condition for $u_{0}$ with account of the adiabaticity criterion (here $\left.b=3 / 4 \pi \nu_{s} u_{t}\right)$ :

$$
u_{0} \leqslant u_{2}(J)=\sqrt{\left(b J^{n / 2}\right)^{2}+u_{t}^{2}\left(1-\frac{3}{8} J\right)^{2}} .
$$

Different areas in plane $u_{0}, J$ are plotted in Fig. 4. Of course, the boundaries between regions are diffused; for example, the particles in area 2 can increase their invariants due to diffusion crossing and then go to «adiabaticity regions».

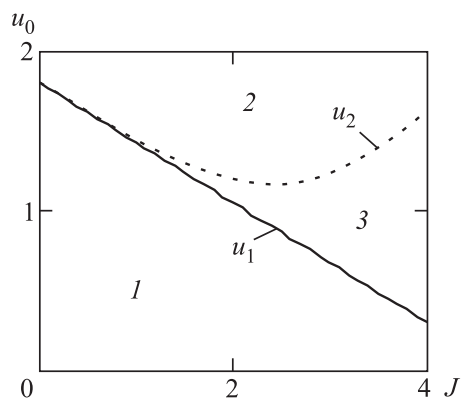

Fig. 4. Separation of different areas in plane $u_{0}, J(n=3, b=0.2)$ : area $1\left(0<u_{0}<u_{1}(J)\right)-$ no resonance crossing; area $2\left(u_{1}(J)<u_{0}<u_{2}(J)\right)$ - adiabatic resonance crossing; area $3\left(u_{2}(J)<\right.$ $\left.u_{0}<\infty\right)$ - diffusion resonance crossing

Beam Losses and Emittance Growth. Let us calculate a number of captured particles. The distribution on amplitudes $u_{0}$ for Gaussian beam is defined by $f\left(u_{0}\right)=u_{0} \exp \left(-\frac{u_{0}^{2}}{2}\right)$; integrating on $u_{0}$ from $u_{1}(J)$ up to $u_{2}(J)$, we obtain the following expression for a number of captured particles:

$$
\frac{\Delta N}{N}=\int_{0}^{(8 / 3) u_{t}} \exp \left[-J-\frac{1}{2}\left(u_{t}-\frac{3}{8} J\right)^{2}\right]\left[1-\exp \left(-\frac{b^{2} J^{n}}{2}\right)\right] d J .
$$

For weak resonance $g \rightarrow 0, \nu_{s}=Q_{s} / g \rightarrow \infty$ and, consequently, $b \rightarrow 0$; then $u_{2}(J) \rightarrow$ $u_{1}(J)$ and the adiabaticity area goes to zero. For strong resonance $\nu_{s}=Q_{s} / g \rightarrow 0$ and, consequently, $b \rightarrow \infty$ (adiabatic limit). Neglecting the term in brackets in RHS of Eq. (21), we obtain that the number of captured particles is

$$
\frac{\Delta N}{N}=\Lambda C(\Lambda)
$$

Here due to Eq. (16)

$$
C(\Lambda)=\int_{0}^{(8 / 3) u_{t}} \exp \left[-J\left(1-\frac{3}{8} u_{t}\right)-\frac{1}{2}\left(\frac{3}{8} J\right)^{2}\right] d J .
$$


For small values of parameter $\Lambda$ we have that $C(\Lambda) \sim 1.3$. The captured particle is lost if its maximal invariant (at the bunch center) exceeds the chamber aperture $J_{\lim }\left(J_{0}(0)>J_{\lim }\right)$. In a frame of our simplified model, we have the following approximate condition of the particle loss: $\frac{2}{\Lambda} \geqslant J_{\text {lim }}, \Delta Q_{\mathrm{sc}}^{0} \geqslant \frac{1}{2} \Delta Q_{0} J_{\text {lim. }}$. If the captured particles are not lost, then the capture results in an increase of the average emittance since part of the synchrotron period is spent by the particle inside the island. The average invariant during the capture period is $J_{\mathrm{av}} \sim \frac{1}{2}\left[J_{0}(0)+J\right]=\frac{1}{\Lambda}+\frac{J}{2}$. Taking into account that the particle spends inside the separatrix only part of period, which is defined by $\mu\left(u_{0}\right)=\frac{2}{\pi} \arcsin \left[\frac{u_{\min }(J)}{u_{0}}\right]$, we find that a change of the average invariant is

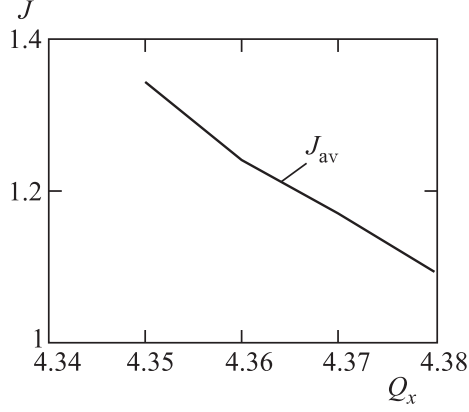

Fig. 5. Dependence of $J$ on $Q_{x}$ $\Delta J_{\mathrm{av}}=\int_{0}^{\infty} \exp (-J)\left(\frac{1}{\Lambda}+\frac{J}{2}\right) d J \int_{u_{1}(J)}^{u_{2}(J)} u_{0} \exp \left(-\frac{u_{0}^{2}}{2}\right) \mu\left(u_{0}, J\right) d u_{0}$.

This integral is calculated numerically for SIS-18; the results are given in Fig. 5 (comparison with data of numerical simulations [1] gives satisfactory coincidence).

\section{CONCLUSIONS}

The analytic approach gives deep insight into the mechanism of the Coulomb resonance crossing; it allows us the straight forward scaling of the parameters for an arbitrary resonance order. Besides, we discover an interesting mechanism of suppression of s.c. losses and the emittance growth using the octupole nonlinearity with correctly chosen sign.

In the future we are going to consider crossing of two-dimensional betatron resonance and to apply the results in analysis of s.c. effects in designed high-current rings: SIS100 (FAIR project, Germany) and TWAC (ITEP, Moscow).

\section{REFERENCES}

1. Franchetti G., Hofmann I. Particle Trapping by Nonlinear Resonances and Space Charge // Nucl. Instr. Meth. A. 2006. V.261. P. 195-202.

2. Zenkevich P.R., Bolshakov A. Ye. Numerical Modeling of Space Charge Influence on the Storage Ring Acceptance // At. Energiya. 2001. V.91, No. 4. P. 294-300.

3. Schoch A. Theory of Linear and Nonlinear Perturbations of Betatron Oscillations in AlternatingGradient Synchrotrons. CERN Report 57-23. 1958.

4. Guignard G. General Treatment of Resonances in Accelerators. CERN Report 78-11. 1978. 
132 Zenkevich P., Hofmann I., Franchetti G.

5. Chao A. W., Month M. Particle Trapping during Passage through a High-Order Nonlinear Resonance // Nucl. Instr. Meth. 1974. V.121. P. 129-138.

6. Keil E. Physics of Colliding Beams. Report CERN/ISR-TH/72-7. 1972.

7. Lichtenberg A., Lieberman M. Regular and Stochastic Mechanics. Springer, 1992. 